This is an electronic reprint of the original article. This reprint may differ from the original in pagination and typographic detail.

Author(s): Laakkonen, Eija; Ronkainen, Paula; Suominen, Harri; Takala, Timo; Koskinen, Satu; Puolakka, Jukka; Sipilä, Sarianna; Kovanen, Vuokko

Title: $\quad$ Muscular Transcriptome in Postmenopausal Women With or Without Hormone Replacement

Year: $\quad 2007$

Version:

Please cite the original version:

Laakkonen, E., Ronkainen, P., Suominen, H., Takala, T., Koskinen, S., Puolakka, J., Sipilä, S., \& Kovanen, V. (2007). Muscular Transcriptome in Postmenopausal Women With or Without Hormone Replacement. Rejuvenation Research, 10(4), 485-500. https://doi.org/10.1089/rej.2007.0536

All material supplied via JYX is protected by copyright and other intellectual property rights, and duplication or sale of all or part of any of the repository collections is not permitted, except that material may be duplicated by you for your research use or educational purposes in electronic or print form. You must obtain permission for any other use. Electronic or print copies may not be offered, whether for sale or otherwise to anyone who is not an authorised user. 


\section{Muscular Transcriptome in Postmenopausal Women with or without Hormone Replacement}

Eija Pöllänen ${ }^{1,2,6}$, Paula HA Ronkainen ${ }^{1,2}$, Harri Suominen ${ }^{2}$, Timo Takala ${ }^{3}$, Satu Koskinen ${ }^{4}$, Jukka Puolakka $^{5}$, Sarianna Sipilä ${ }^{1,2}$, Vuokko Kovanen ${ }^{2}$

${ }^{1}$ Finnish Centre for Interdisciplinary Gerontology, University of Jyväskylä, Jyväskylä, FIN40014, Finland; ${ }^{2}$ Department of Health Sciences, University of Jyväskylä, Jyväskylä, FIN40014, Finland; ${ }^{3}$ Department of Sports Medicine, Deaconess Institute, Oulu, FIN-90100, Finland; ${ }^{4}$ Institute of Sports Medicine, Bispebjerg University Hospital, Copenhagen, DK-2400, Denmark; ${ }^{5}$ Central Finland Central Hospital, Jyväskylä, FIN-40620, Finland

Word count:

${ }^{6}$ Address for correspondence and reprints:

$\begin{array}{ll}\text { Author: } & \text { Eija Pöllänen } \\ \text { Address: } & \text { PO Box 35/Viveca (Rautpohjankatu 8) } \\ & \text { FIN-40014 University of Jyväskylä } \\ & \text { FINLAND } \\ & +358-14-2604531 \\ \text { Phone: } & +358-14-2604600 \\ \text { Fax: } & \text { eija.pollanen@sport.jyu.fi }\end{array}$

Keywords: menopause, hormone replacement therapy, sarcopenia, muscular transcriptome Abbreviated title: Muscle Transcriptome in Postmenopausal Women 


\section{ABSTRACT}

The loss of muscle mass and strength with aging is well characterized, but our knowledge of the molecular mechanisms underlying the development of sarcopenia remains incomplete. Although menopause is often accompanied with first signs of age-associated changes in muscle structure and function, the effects of hormone replacement therapy (HRT) or menopause-related decline in estrogen production in the muscles of postmenopausal women is not well understood.

Furthermore the knowledge of the global transcriptional changes that take place in skeletal muscle in relation to estrogen status has thus far been completely lacking. We used a randomized double-blinded study design together with an explorative microarray experiment to characterize possible effects of continuous, combined HRT and estrogen deprivation on the skeletal muscle of fifteen women. Here, we report the differential response of both GO-annotated biological processes and some individual genes responding differentially to the use or non-use of HRT. Our results revealed transcription level changes in, e.g., muscle protein and energy metabolism. In particular, the ubiquitine-proteosome system was found to be effected at several levels. HRT seemed to partially counteract the postmenopause-related transcriptional changes. Our results suggest that during the early postmenopausal years, when there is no counteracting medication available, muscle transcriptome changes notably, whereas HRT appears to slow-down this phenomenon and could therefore aid in maintaining proper muscle mass and function after menopause. 


\section{INTRODUCTION}

Even physically active, healthy persons confront sarcopenia, i.e., aging-related gradual loss of muscle mass and strength. Although these changes in muscle structure and function are well documented in scientific literature, ${ }^{1-3}$ our understanding of the molecular mechanisms underlying the development of sarcopenia remains incomplete. There are clear gender specific differences in the prevalence of sarcopenia. In women, deterioration in muscle performance has been observed as early as during perimenopause, ${ }^{4}$ making them more vulnerable to sarcopenia than agematched men. ${ }^{5,6}$ This indicates that the female sex hormones, estrogens, might be involved in the early developmental steps of sarcopenia.

The current understanding concerning the functions of estrogens is still fragmentary. Many classical effects of estrogens, both in reproductive and in non-reproductive tissues, are thought to be mediated via their nuclear receptors, ESR1 and ESR2, which directly or indirectly regulate the transcription of many genes. ${ }^{7}$ Estrogens also act through cell membrane located receptors, which can be independent of ESRs. ${ }^{8}$ ESRs have been found in human skeletal muscle ${ }^{9,10}$ suggesting the contribution of estrogens to muscle functions. Recent experimental animal studies have provided evidence that estrogen, via its receptors, has a role in glucose metabolism in muscle ${ }^{11,12}$. The gathering information points out that estrogen signaling may have fundamental implications for muscle performance and therefore could partially explain the demonstrated increase in the loss of muscle mass and strength upon menopause.

Postmenopausal state with nearly undetectable ovarian estrogen production offers an opportunity to study the effects of estrogen signaling on muscle functions. Some of the randomized, controlled trials in postmenopausal women have shown increased muscle mass 
and/or strength after hormone replacement therapy (HRT) ${ }^{13-16}$ whereas others have failed to show any benefit from HRT in the prevention of sarcopenia. ${ }^{17-19}$ A partial explanation for this discrepancy lies in differences in methodology and subjects' characteristics.

To our knowledge, most studies conducted thus far have focused on the effects of estrogen deprivation or replacement on the physical parameters of muscles, but a transcriptome-wide analysis of estrogen deprivation or HRT-induced changes in the skeletal muscle of postmenopausal women has not till now been undertaken. Therefore, we used needle biopsy samples from the vastus lateralis muscle of healthy, early postmenopausal women, to establish a general view on the alterations occurring in the muscular transcriptome during postmenopause with and without HRT. 


\section{MATERIALS AND METHODS}

\section{Study design and measurements}

This study is part of a larger randomized, placebo-controlled experiment on bone and muscle structure and function in relation to exercise and hormone replacement therapy. ${ }^{14}$ The study sample was taken from postmenopausal women aged 50-57 years living in the city of Jyväskylä, Finland. Initially 118 women eligible for the study were invited for a clinical examination to assess hormonal menopausal status via serum steroid measurements (DELFIA, Wallac). Eighty women were then randomized into four study groups: exercise, exercise+HRT, HRT and non-HRT. To be eligible for the trial, participants had to have no serious medical conditions, no current or previous use of medication including estrogen, fluoride, calcitonin, biophosphonates or stereoids, last menstruation at least 0.5 years but no more than 5 years ago, FSH level above $30 \mathrm{IU} / \mathrm{l}$, and no contra-indications for exercise and HRT. The HRT intervention was carried out double blinded. All the participants took placebo or the combined estradiol (2 $\mathrm{mg}$ ) and noretisterone acetate $(1 \mathrm{mg})$ product (Kliogest ${ }^{\circledR}$; Novo Nordisk), one tablet per day, for one year. The study reported here focuses on the effects of menopause, with and without HRT (the HRT and non-HRT groups) in postmenopausal women. Being out of the scope of this research, the exercise and exercise+HRT groups were excluded from analysis.

Phenotypes were measured, hormonal status quantified and needle muscle biopsies obtained at baseline and after one year in the original study. Lean body mass (LBM) and body fat mass were assessed using bioelectrical impedance (Spectrum II, RJL Systems). Computed tomography (CT) scans were obtained from the thigh muscles on the side of the dominant hand using a Siemens Somatom DR scanner (Siemens AG). CT scans were analyzed using BonAlyse 
1.0 software (Commit Ltd) to measure lean tissue cross-sectional area of the quadriceps femoris (qCSA). Muscle biopsies were obtained from mid-part of vastus lateralis defined as a midpoint between the greater trochanter and the lateral joint line of the knee. In order to avoid variation due to sampling, the biopsy protocol was standardized within the study. Biopsy samples (average $60 \mathrm{mg}$ ) were snap frozen in liquid nitrogen after removing all visible residues of fat and connective tissue and stored in $-80{ }^{\circ} \mathrm{C}$ until used. In the present study we used baseline and follow-up samples and measurements from ten subjects in the HRT group and five subjects in the non-HRT group from whom the biopsies were available.

The study was carried out in accordance with the Declaration of Helsinki (1989) of the World Medical Association, and was approved by the ethics committee of the Central Finland Central Hospital. An informed consent was given by all subjects.

\section{RNA preparation and cRNA generation}

Frozen biopsy samples were homogenized in Trizol-reagent (Invitrogen) with the aid of a FastPrep FP120 apparatus (MP Biomedicals). Total RNA was isolated according to the manufacturer's protocol. The RNA concentration was measured spectrophotometrically. RNA quality was determined using an Experion ${ }^{\mathrm{TM}}$ (Bio-Rad Laboratories), which enables assessment of both integrity and purity of RNA. Only good pure, quality RNA was used in further analysis (260/280 ratio 1.8). An Illumina RNA amplification kit (Ambion) was used according to the manufacturer's instructions to obtain biotin-labeled cRNA for the microarray hybridizations. 500 ng of total RNA was used in each amplification protocol. Experion ${ }^{\mathrm{TM}}$ was used to perform quality control after amplification. The quality of the samples was further tested with the Illumina protocol to obtain biotin-labeled cRNA, and the test hybridizations were performed 
using the Sentrix-16 BeadChips (BD-15-101, Illumina) with Human Sampler probe set for 517 genes. TestChip data has been deposited in the public Gene Expression Omnibus (GEO) database (http://www.ncbi.nlm.nih.gov/geo/) under series code GSE6378.

\section{Microarray experiments}

Hybridization onto the Sentrix HumanRef-8 Expression BeadChips (BD-25-201, Illumina) as well as washing and scanning were performed according to the Illumina BeadStation 500x manual (revision C). We used five 8-sample BeadChips to hybridize a total of 30 samples. Both baseline and follow-up samples for each study subject were hybridized onto the same BeadChip. The hybridization mix was prepared by combining $125 \mu$ l of hybridization buffer with $75 \mu 1$ formamide. The hybridization mix $(22.7 \mu \mathrm{l})$ was added to each $0.85 \mu \mathrm{g}$ of labeled cRNA and the resulting assay solution $(34 \mu \mathrm{l})$ was preheated at $65^{\circ} \mathrm{C}$ for $5 \mathrm{~min}$. Adhesive release liner from BeadChip was removed and the assay sample was then applied onto the center of each array. Cap was aligned on top of the hybridization cartridge and closed with a clamp. The hybridization cartridges were attached to the BeadChip hybridization wheel with rotation in a hybridization oven at $55^{\circ} \mathrm{C}$ for an additional $16-20$ hours.

The hybridization cartridges were disassembled: the cover seals were removed following immediate submerging of the BeadChips in the wash solution and incubation for $15 \mathrm{~min}$ on an orbital shaker at room temperature. The slide rack was then transferred to staining dish containing $100 \%$ ethanol and incubated on an orbital shaker for $10 \mathrm{~min}$. The slides were rinsed in fresh wash solution for 2 min on an orbital shaker. Blocking was performed in $4 \mathrm{ml}$ of Block buffer in disposable wash trays, which were incubated for 10 minutes on an orbital shaker. Staining was performed in 1:1000 dilution of Cy3-conjugated streptavidin $(1 \mathrm{mg} / \mathrm{ml})$ in Block 
buffer for 10 min on an orbital shaker. The slides were transferred to a slide rack in Wash solution and incubated on orbital shaker for $5 \mathrm{~min}$. The racks were dried by centrifugation with plate holders at $275 \mathrm{rcf}$ for $4 \mathrm{~min}$ at room temperature.

The slides were scanned by a confocal laser scanning system (Illumina BeadReader Rev. C) supporting $\mathrm{Cy} 3$ and $\mathrm{Cy} 5$ imaging and performing with 0.8 microns resolution. The data were acquired by BeadStudio Direct Hybridization V.1.5.0.34. Gene expression data and the raw data sets are publicly available in the GEO database under the series code GSE6348.

\section{Data analysis}

The effects of HRT on phenotypes were tested using analysis of variance for repeated measures (ANOVA).

Sentrix HumanRef-8 Expression BeadChip contains probes for 24000 NCBI RefSeq-genes and on average 30 beads to each probe. The signal for individual genes is determined by calculating the average signal intensity, excluding beads that fall outside three normal standard deviations of median intensity. After this hybridization quality control, the high quality signals were quantile-normalized in order to perform cross-chip analysis. Median normalization was also inspected and found to give results similar to those of quantile normalization. All data were filtered with a background value such that upregulated genes had to be above the background value in follow-up samples and downregulated genes above the background value in baseline samples. Each BeadChip contains 778 different negative control probes, with no match to the human genome. There are on average 30 beads to each negative control probe. The individual background value for each BeadChip is determined by averaging the signal intensities of the negative control probes. 
To be recognized as up- or downregulated, in users and non-users of HRT, the gene had to meet the following criteria: the change between follow-up and baseline condition had to be $>0$ (upregulated genes) or $<0$ (downregulated genes), the change had to be into the same direction in all but one subject and, finally, the change had to be significantly different between the HRT and non-HRT groups. The difference between study groups was statistically identified using ttest for independent samples with a threshold $p$-value $\leq 0.05$. The t-test was adjusted for multiple comparisons using the Benjamini-Hochberg false discovery rate (FDR) correction. ${ }^{20}$ Genes with FDR-corrected $\mathrm{p} \leq 0.05$ were considered differentially expressed. Our approach maximizes the stringency in avoiding the false positive error in the data analysis, but allows minor variability between study subjects. Literally this means that $90 \%$ of the subjects in the HRT group and 80 $\%$ of the subjects in the non-HRT group needed to have the same directionality in the gene expression before the gene was taken into account in the data analysis. Due to the limited number of samples from the non-HRT subjects it was not possible to be as stringent in the nonHRT group as in the HRT group. Identical analyses were done separately for the HRT and nonHRT groups. SPSS 12.0.1, R 2.3.1/Bioconducter (www.r-project.org, www.bioconductor.org) and Inforsense KDE 3.1 software were utilized for data analysis.

The hierarchical clustering was performed with Cluster. ${ }^{21}$ Genes were clustered by correlation uncentered similarity metric using single linking clustering. Figures were generated with Maple Tree (http://rana.lbl.gov/EisenSoftware.htm).

\section{Ontology enrichment}

The lists of genes significantly up- or downregulated in the HRT and the non-HRT groups (FDR corrected $\mathrm{p} \leq 0.05$ ) were searched for possible enrichment in certain biological processes 
defined by Gene Ontology (GO) annotation association. ${ }^{22}$ For this analysis we used Web-based ONTO-Express software (http://vortex.cs.wayne.edu/projects.htm). ${ }^{23,24}$ The number of genes corresponding to each GO-category among the differentially expressed genes in the HRT and non-HRT groups was compared with the number of genes expected for each GO-category on the basis of their representation on the HumanRef- 8 BeadChip. Significant differences from the expected numbers were calculated using a two-sided binomial distribution with FDR-correction. Only GO-categories with FDR-corrected p-values $\leq 0.05$ were reported.

\section{Real Time RT-PCR}

We used commercial TaqMan gene expression assays (Applied Biosystems) to verify the microarray results for eight selected genes (Ar: Hs00171172_m1, Fbxo11: Hs00251516_m1, Fbxo32:Hs00369714_m1, Usp1:Hs00163427_m1, Usp2:Hs00899199_g1, Usp15: Hs00378613_m1, Usp50:Hs01596824_m1, Ogt:Hs00269228_m1). The levels of expression of Esr1 and Esr2 were also determined by TaqMan gene expression assays (Hs01046812_m1 and Hs01100358_m1, respectively). The expression of Mgea5 was verified by TaqMan gene expression assay Hs00201970_m1. One $\mu \mathrm{g}$ of RNA, originated from the same muscle samples as used in microarray experiments, was reverse transcripted into cDNA and used in Real Time RTPCR assays according to the instructions provided by the manufacturer (Applied Biosystems). Assays were performed with an Applied Biosystems ABI 7300 unit using standard PCR conditions recommended by manufacturer. Each sample was run in triplicate and compared against the reference sample. The reference sample was constructed by combining an equal amount of each sample in a single tube. In our hands Gapdh performed best of the three control transcripts tested (Gapdh: Hs99999905_m1, 18S: Hs99999901_s1, Ddx52: Hs00294711_m1) 
and was therefore used as an endogenous control. We evaluated both the comparative $\mathrm{C}_{\mathrm{T}}$ method $^{25}$ and sigmoidal curve-fitting (SCF) method ${ }^{26}$ for their ability to provide reliable results for gene expression. In our hands, both the comparative $\mathrm{C}_{\mathrm{T}}$ and $\mathrm{SCF}$ methods had a very similar intra-assay coefficient of variation, $6.6 \%$ and $6.2 \%$, respectively. The SCF method was used to present the Real Time RT-PCR results in the further analyses. Pearson correlation coefficient calculated with SPSS 12.0.1 software was used to determine the correlation between the microarray and Real Time RT-PCR results. 


\section{RESULTS}

\section{Phenotypic results}

All the subjects were postmenopausal at the baseline. The average age of the subjects was $53.5 \pm 1.5$ in the HRT and 53.7 \pm 2.4 in the non-HRT group and their last menarche had been 2.5 \pm 2.4 and 3.3 \pm 5.3 years ago, respectively. The postmenopausal status was further confirmed by low estrogen levels and high serum follicle stimulating hormone (FSH) levels (Table 1) at the baseline. As expected the estrogen levels rose in the HRT group with a concomitant decrease in FSH levels, showing the efficacy of the HRT used. The levels of sex hormone-binding globulin (SHBG), which is the most important estrogen and testosterone binding protein in serum, declined in the HRT group and increased in the non-HRT group. No differences were observed in testosterone levels between the study groups throughout the intervention.

As shown in Table 2, no statistically significant changes occurred in total body fat mass. However, LBM increased during HRT, while it decreased in the non-HRT group. Also qCSA increased in the HRT group and remained unchanged in the non-HRT group.

\section{Data mining for differentially regulated genes in the HRT and non-HRT groups}

The data mining procedure is presented in Figure 1. Fifteen muscle biopsies were used as a source for RNA. Labeled cRNA samples were hybridized onto HumanRef- 8 BeadChips, which provides transcriptional coverage of 24000 well-characterized, human RefSeq-genes. Among these transcripts we first evaluated which genes were either up- or downregulated during the intervention within a study group before statistical between-group-tests. We used FDRcorrection to control false positive error and report only the results with FDR-corrected $p \leq 0.05$. 
According to this criterion, we found changed expression for 97 genes ( 64 up, 33 down) in the HRT group. From these genes, seven were significantly differentially expressed in the HRT group when compared to the non-HRT group. Identical analysis was done for the non-HRT group, revealing changed expression of 4195 genes (2138 up, 2057 down) from which 214 (139 up, 75 down) were significantly differentially expressed when compared to the expression of same genes in the HRT group. The hierarchical clustering of HRT- and non-HRT-specific genes is presented in Figure 2. In further analyses, we used these gene lists to investigate possible enrichment of biological processes before conducting detailed gene-by-gene analyses.

\section{Significantly enriched gene ontology categories}

To be able to characterize which biological processes are affected by HRT or postmenopause in skeletal muscle, we used web-accessible ONTO-Express ${ }^{23,24}$, which enables classification of genes according to their involvement in various biological processes defined by GO-annotation. ${ }^{22}$ In this analysis we used the lists of genes found to be significantly differentially expressed between the HRT and the non-HRT groups. Only $20 \%$ of these genes were significantly located in the GO-annotated biological process while the majority was randomly spread through the different categories. Only the categories with significant hits, certified by FDR-corrected $\mathrm{p} \leq 0.05$, are reported. These hits could be assigned into four broad categories: "RNA metabolism", "protein posttranslational modifications", "cellular catabolic processes" and "other biological processes" (Table 3). Each of these categories includes several GO-annotated classes from which "carbon utilization" and "catecholamine metabolism" are the most significant and "proteolysis and peptidolysis" is the largest with altogether 15 genes. Other 
large, significant classes with 3-6 genes were "RNA splicing", "protein folding" and "protein amino acid glycosylation”.

\section{The mRNA levels of estrogen receptors are not affected by HRT in skeletal muscle}

Since the expression of both Esrs has been shown to respond to the availability of estrogen

${ }^{27}$ we were a bit surprised not to find them among the significantly regulated genes in the HRT group. The change in the expression of Esrl was inconsistent and did not follow the availability of estrogen. In most subjects the expression of Esr2 did not exceed the background value. Both results were confirmed by Real Time RT-PCR.

\section{Genes specifically responding to the use of HRT}

Table 4 and Figure 2C summarize the genes, which we found to be significantly up- or downregulated in the HRT group when compared to the non-HRT group. $O$-linked Nacetylglucosamine (GlcNAc) transferase $(O g t)$ was found to be upregulated by six percent in the HRT group. This increment is, however, five times less than that observed in the non-HRT group. $O g t$ has been shown to catalyze nucleoplasmic glycosylation of serine or threonine residues. ${ }^{28,29}$ Since both phosphorylation and glycosylation compete for the same sites as demonstrated for ESRs, MYC and SP1 transcription factors, and other important regulatory proteins, it has been proposed that alternating glycosylation/phorphorylation may serve as a switch between inactive and active protein forms. ${ }^{30}$

Recently, Whisenhunt et al. ${ }^{31}$ showed by in vitro experiments that OGT forms a bifunctional repression-complex with nuclear/cytoplasmic $O$-GlcNAcase and acetyl transferase (NCOAT), which has formerly been called MGEA5. In this complex, NCOAT can rapidly 
reverse OGT-induced $O$-GlcNAcylation as well as deacetylation induced by histone deacetylases. In addition, the absence of a functional NCOAT was shown to lead to failure in removing the $O$-GlcNAc modification, which in turn was sufficient to inhibit the expression of estrogen responsive genes. To be able to evaluate whether OGT/NCOAT-repression-complexes can be formed in the muscles of postmenopausal women we analyzed the expression pattern of Ncoat by Real Time RT-PCR. This analysis verified the results of the microarray study and showed that both $O g t$ and Ncoat are indeed expressed in muscle tissue and their expression is strongly correlated $(r=0.724, p<0.001)$ and coincided $\left(r^{2}=0.524\right)$. Thus it is possible that OGT/NCOAT-repression-complex-mediated regulation can occur in the muscles of postmenopausal women.

Among the HRT-responding genes we also found genes encoding both catabolic and anabolic agents. Androgen receptor (Ar) was significantly upregulated (13\%) in the HRT group. Ar represents an anabolic agent which is known to mediate androgen signaling, ${ }^{32}$ leading to increased muscle strength and LBM. ${ }^{33-35}$ In the HRT group, we also found significant upregulation of a catabolic agent, Fbxo32, also known as Atrogin-1, which has been shown to be highly expressed during muscle atrophy. ${ }^{36,37}$

The other genes found to respond to the use of HRT in our study were Rnaseh2c, a potential ribonuclease, Sult1al, a member of the sulfotransferase family, Il4il, an interleukin 4induced 1 gene and Rab31, a member of the RAS oncogene family.

\section{Up- and downregulated genes during early postmenopause in the non-HRT group}


Complete lists of up- and downregulated genes in the non-HRT group are shown in Supplementary Tables 1 and 2 and Figure 2A and B. Examples of the non-HRT group specific genes are presented in Table 5 and reviewed in the following section.

Ogt, which was found to be moderately upregulated in the HRT group, was even more extensively upregulated in the non-HRT group (30\%) suggesting that upregulation in the expression of $O g t$ is related to postmenopause and that HRT partially suppresses this upregulation. The other gene, whose transcription was found to be significantly differentially regulated in both group comparisons, was $A r$. However, in this case the direction of regulation in the HRT and the non-HRT groups was inverted (Tables 4 and 5), suggesting the contribution of estrogen status to the regulation of $A r$ at the mRNA level.

The gene family encoding ubiquitin-specific peptidases (USPs) had four members among the differentially regulated genes in the non-HRT group when compared to the HRT group. Usp1 and $U s p 15$ were upregulated (16\% and $24 \%$, respectively) and $U s p 2$ and $U s p 50$ downregulated (-12\% and $-10 \%$, respectively). USPs are known to cleave the ubiquitin tail from proteins targeted to degradation via the ubiquitin-proteosome system. ${ }^{38,39} \mathrm{We}$ also found other components of the ubiquitin-proteosome system to be differentially regulated during postmenopause. Ubiquitin-conjugating enzyme, $U b e 2 g 2$, was downregulated by five percent and ubiquitin protein ligase, Fbxo11, upregulated by 14 percent.

\section{Evaluation of technical and biological validity of microarray results}

Five randomly selected cRNA samples were hybridized onto the TestChip containing 517 genes. From these genes 433 were detected in our samples. From the expressed genes we selected those with identical probes in both the HumanRef-8 BeadChip and TestChip; 378 
identical probes were found. We used this set of probes to evaluate the technical validity of our microarray results by Pearson correlation analysis. The correlation between the gene expression values for five replicated samples was high $(r=0.97, p<0.001)$, verifying that the technical reproducibility of our experiment was significant $\left(r^{2}=0.94\right)$.

To be able to assess the biological validity of our microarray results, we chose to use Applied Biosystems's TaqMan gene expression assays for eight genes with probes representing different regions of the studied transcripts than present in the HumanRef- 8 BeadChip. Ogt and $A r$ were selected on the basis of their high significance among the upregulated genes in the HRT group (FDR-corrected p-values 0.001 and 0.009 , respectively). We also wanted to evaluate highly related genes Usp1, Usp2, Usp15 and Usp50 as well as Fbxol1 and Fbxo32. The expression level of Usp50 was too low for reliable detection with Real Time RT-PCR. We were, however, able to detect the other genes and the results were in good agreement with the microarray results (Figure 3), the average correlation coefficient being 0.53 . This association is rather high considering that in the HumanRef-8 BeadChip the probes are designed for 3 'prime untranslated regions and, in the Taqman gene expression assays, for coding regions spanning the exon-intron boundaries. By representing different regions of the same transcript, these two methods might recognize somewhat different pools of the same transcript. For example, the microarray result may be representative of the initial heterogeneous nuclear RNA or pre-mRNA while Real Time RT-PCR may be representative of more processed, i.e., spliced mRNA.

In conclusion, both the technical and biological validity of our microarray results was significant. 


\section{DISCUSSION}

To our knowledge this is the first attempt to use explorative microarray analysis to identify the changes that take place in the skeletal muscle of postmenopausal women in relation to the hormonal status. High density oligonucleotide microarrays have previously been utilized to characterize, e.g., age- or exercise-related changes in human skeletal muscle, ${ }^{40-49}$ but not directly menopause- or HRT-related transcriptional changes. The transcriptome-wide effects of HRT have only been studied in blood samples from postmenopausal women. ${ }^{50}$ Therefore, our study is a novel opening to a study line investigating the molecular mechanisms behind the physical impairments that may begin already during the early postmenopausal years.

According to our findings, during the first postmenopausal years the muscle transcriptome changes markedly. At the individual gene level more alterations were observed in the muscles of women not using HRT than in the muscles of HRT users. HRT appears to balance these transcriptional modulations and therefore could reduce or slow-down the age-related changes in the muscular transcriptome. The GO analysis revealed that, e.g., "RNA metabolism", "protein posttranslational modifications" and "cellular catabolic processes" were affected by postmenopausal status with or without HRT. The most noteworthy finding was that many genes related to "proteolysis and peptidolysis" were affected by postmenopause, but this effect was not seen after the use of HRT.

From "proteolysis and peptidolysis"-related genes we found four ubiquitin proteases (Usp1, Usp2, Usp15, Usp50), one ubiquitin-conjugating enzyme (Ube2g2) and one ubiquitin protein ligase (Fbxol1) to be differentially expressed in the non-HRT group when compared to the HRT group. In the HRT group we found significant upregulation of another protein ligase, Fbxo32, which is better known as Atrogin-1. In experimental animal studies, Atrogin-1 has been shown to 
be highly expressed in skeletal muscles which were atrophying due to fasting or diverse diseases. ${ }^{36,37}$ In very recent studies, downregulation of ATROGIN-1 in sarcopenic, slow-twitch muscles of aged, female rats was demonstrated ${ }^{51}$ whereas opposite results were obtained in fasttwitch muscles of aged, male rats. ${ }^{52}$ These studies suggest that the association between Atrogin-1 and muscle atrophy may be fiber type- or gender-dependent. Another explanation could be that sarcopenia is mechanistically different from acute muscle atrophy, which is marked by increased expression of Atrogin-1, as suggested by Edstrom et al. ${ }^{51}$ This statement is supported by the findings of Giresi and colleagues, ${ }^{45}$ who conducted a microarray study with muscle samples from 19-25-yr-old and 70-80-yr-old male subjects to identify the molecular signature of sarcopenia. The identified signature did not include Atrogin-1. Also our results testify against the role of Atrogin-1 in the development of sarcopenia, since the same study population (HRT group) showed a significant increase in the expression of Atrogin-1, in qCSA and in LBM (Table 2), suggesting that use of HRT may favor anabolic more than catabolic reactions.

The other genes encoding proteolysis-related proteins and found to be affected by postmenopause were: plasminogen activator, Plau, ring finger proteins, Rnf13 and Rnf24, endoplasmic reticulum degradation enhancer, Edem3, carboxypeptidase D, Cpd, apoptosisrelated cysteine peptidase, Casp9, dipeptidylpeptidase, Dpp6 and macrophage elastase, Ela2. It has also been suggested that the multifunctional OGT, whose gene expression was found to be increased by 30 percent in the muscles of non-HRT users and by only six percent in the muscles of HRT users, regulates the activity of the proteosome system. OGT catalyses transport of $O$ GlcNAc to numerous substrates. ${ }^{53} O$-GlcNAcylation of RPT2, one of the proteosomal ATPases, by OGT has been shown to inhibit the activity of the proteosome system. ${ }^{54}$ Our results do not permit us to say whether the net sum of all the observed proteosome system-related 
transcriptional changes will generate increased or decreased proteolysis in the muscles of postmenopausal women; however, we can conclude that menopause has a clear effect on the ubiquitin-proteosome machinery, which might affect the muscular protein turn-over in many ways.

In our study the reduction in the expression of $A R$ coincides with the mean decrease in LBM in the non-HRT group. It remains to be seen whether this is a direct or indirect effect of minimal estrogen production after menopause. Among HRT users, the expression of AR increased concomitantly with the mean increase in LBM. The HRT product utilized was a combination of $17 \beta$-estradiol and noretisterone acetate, which was administered continuously. Since noretisterone acetate may act as a weak androgen and androgens have been shown to both up- and downregulate the expression of $\mathrm{AR},{ }^{55}$ it is not clear whether estrogen itself or noretisterone acetate induced the observed enhancement of AR expression among the HRT users. A model of estrogen function has been proposed in which estrogen may exert its effects through membrane-associated ESRs, through AR or through another as yet unidentified estrogen-binding molecule in addition to its nuclear receptors. ${ }^{56}$ In our experiment, the expression of $E s r 2$ was below the level of detection and the expression of Esrl was not consistent with the use of HRT. Unfortunately, due to the limited amount of muscle samples we were unable to measure ESR1, ESR2 or AR expression at protein level, thus leaving open the question of the availability of these receptor proteins. Therefore, it remains possible that ESR1 or ESR2 proteins are present in muscle tissue enabling them to participate in HRT-mediated signaling. Alternatively the signal could be delivered through AR or some other receptor molecule. Our finding that the mRNA levels of Esrl and $E s r 2$ were not regulated by HRT is in line with recent results from animal studies. Barros et al. ${ }^{11}$ showed that aromatase-knockout, 
male mice, lacking estrogen production completely, nevertheless expressed both ESR1 and ESR2 proteins in muscle cells, demonstrating that estrogen itself does not determine the expression of its receptors in muscle.

Recent results suggest that estrogen signaling may have fundamental implications for the age-related increase in the deterioration in muscle strength and also in the experience of muscular fatigue during physical activity. Muscle is a highly glucose-sensitive tissue and therefore the maintenance of glucose homeostasis is one of the major determinants of muscle performance. Estrogen signaling has been shown to affect glucose homeostasis and insulin sensitivity ${ }^{57-59}$ although the mechanisms are not understood. The uptake of glucose into muscle cells is mainly mediated by glucose transporter 4 (GLUT4), which can be activated by insulinand/or exercise-induced signal transduction. ${ }^{60}$ In both animal and human studies estrogen has been shown to modulate insulin action by affecting insulin secretion ${ }^{61}$ and to participate in the regulation of GLUT4 expression in the skeletal muscle of mice. ${ }^{12}$ Interestingly, the overexpression of OGT, which we found to be highly increased at the mRNA level upon menopause, has also been shown to induce insulin resistance. ${ }^{62}$ The functionality of OGT is directly dependent on the availability of glucose. When glucose fluxes into muscle cells, OGT is able to use it as a substrate in order to regulate the activity of many transcription factors and also the activity of the ubiquitin-proteosome system. This in turn changes the rate of protein degradation, thereby affecting the availability of free amino acids and the need for glucogenesis. The results presented in this paper as well as results from the other studies described above suggest that estrogen status has a versatile role in muscle energy metabolism and, therefore, menopause with estrogen deprivation may influence the rate of deteriorations observed in aging muscles. 
The relatively small study sample size in this study may affect the results obtained. However, similar ${ }^{41,43,45,47,63}$ or even smaller ${ }^{40,42,44,46,48}$ numbers of subjects have been successfully utilized in previous microarray studies investigating transcriptional changes in skeletal muscle. In our study, an extra strength is achieved by using RCT-design with repeatedmeasures analysis, which enables the detection of intra-individual changes in gene expression. The purpose of our study was to determine the long-term effects of the usage or non-usage of HRT; therefore we were not surprised to detect lower changes in the gene expressions than seen in studies investigating the acute effects. With our approach, in which the observed changes in the gene expression are demanded to be consistent within a study group with extra emphasis in reducing the false positive error especially in the HRT group, we are confident that the detected changes in the gene expression are of biological relevance and worth taken into consideration when describing the phenomena under investigation. Even so the observed results should always be confirmed with a different experimental setup from which a great example is the study of McClain and colleagues. ${ }^{62}$ They showed that even a modest, 20 percent, overexpression of OGT in fat and muscle tissue leads to insulin resistance and hyperleptinemia in transgenic mice which, however, may not be directly applicable in humans.

In conclusion, we used a randomized double-blinded study design together with explorative microarray experiment to characterize the possible effects of menopause with or without hormone replacement on the skeletal muscles of early postmenopausal women. Our study revealed more transcriptional alterations in the muscles of women not using HRT than in the muscles of HRT users. In particular the muscle protein and energy metabolism were affected. HRT seemed to partially counteract these changes. We have previously reported, in a larger study with the same study subjects, that leg extensor power, assessed by vertical jumping height, 
and thigh muscle mass increased upon the use of HRT compared to the non-HRT group, where they decreased. ${ }^{14}$ Parallel results were obtained with the smaller study sample used in microarray analysis, albeit the functional measurements did not reach statistical significance. Together these results suggest that during early postmenopause, when there is no counteracting medication available, the muscle transcriptome shows notable change; this, in turn, may be related to the deterioration in muscle characteristics in later life. HRT appears to balance or slow-down this phenomenon and could therefore aid in maintaining proper muscle mass and function after menopause. However, in the light of the HRT-related risks reported in the Women's Health Initiative ${ }^{64}$ and Estonian Postmenopausal Hormone Therapy trial ${ }^{65}$, the use of HRT should always be carefully considered and individual's family history and personal risk factors taken into account. 


\section{ACKNOWLEDGEMENTS}

We thank personnel at the Turku Centre for Biotechnology, especially Annika Brandt, Marjo Linja, Stephen Rudd and Lilli Saarinen for conducting the microarray hybridizations and for aid in the initial data analysis. Jarno Tuimala at the Finnish IT Center for Science (CSC) is thanked for useful discussions and comments on statistical questions. Urho Kujala (Department of Health Sciences, University of Jyväskylä) is acknowledged for critical reading and valuable comments on the earlier drafts of this article. This work was supported by grants from the Finnish Ministry of Education and from the Jenny and Antti Wihuri Foundation. 


\section{REFERENCES}

1. Jackman RW, Kandarian SC. The molecular basis of skeletal muscle atrophy. Am J Physiol Cell Physiol. 2004; 287(4):C834-43.

2. Nair KS. Aging muscle. Am J Clin Nutr. 2005; 81(5):953-963.

3. Degens H, Alway SE. Control of muscle size during disuse, disease, and aging. Int J Sports Med. 2006; 27(2):94-99.

4. Calmels P, Vico L, Alexandre C, Minaire P. Cross-sectional study of muscle strength and bone mineral density in a population of 106 women between the ages of 44 and 87 years: Relationship with age and menopause. Eur J Appl Physiol Occup Physiol. 1995; 70(2):180186.

5. Phillips SK, Rook KM, Siddle NC, Bruce SA, Woledge RC. Muscle weakness in women occurs at an earlier age than in men, but strength is preserved by hormone replacement therapy. Clin Sci (Lond). 1993; 84(1):95-98.

6. Samson MM, Meeuwsen IB, Crowe A, Dessens JA, Duursma SA, Verhaar HJ. Relationships between physical performance measures, age, height and body weight in healthy adults. Age Ageing. 2000; 29(3):235-242.

7. Hewitt SC, Deroo BJ, Korach KS. Signal transduction. A new mediator for an old hormone? Science. 2005; 307(5715):1572-1573. 
8. Revankar CM, Cimino DF, Sklar LA, Arterburn JB, Prossnitz ER. A transmembrane intracellular estrogen receptor mediates rapid cell signaling. Science. 2005; 307(5715):16251630.

9. Lemoine S, Granier P, Tiffoche C, Rannou-Bekono F, Thieulant ML, Delamarche P. Estrogen receptor alpha mRNA in human skeletal muscles. Med Sci Sports Exerc. 2003; 35(3):439-443.

10. Wiik A, Glenmark B, Ekman M, Esbjörnsson-Liljedahl M, Johansson O, Bodin K, Enmark E, Jansson E. Oestrogen receptor beta is expressed in adult human skeletal muscle both at the mRNA and protein level. Acta Physiol Scand. 2003; 179(4):381-387.

11. Barros RP, Machado UF, Warner M, Gustafsson JA. Muscle GLUT4 regulation by estrogen receptors ERbeta and ERalpha. Proc Natl Acad Sci U S A. 2006; 103(5):1605-1608.

12. Barros RP, Machado UF, Gustafsson JA. Estrogen receptors: New players in diabetes mellitus. Trends Mol Med. 2006; 12(9):425-431.

13. Skelton DA, Phillips SK, Bruce SA, Naylor CH, Woledge RC. Hormone replacement therapy increases isometric muscle strength of adductor pollicis in post-menopausal women. Clin Sci (Lond). 1999; 96(4):357-364.

14. Sipila S, Taaffe DR, Cheng S, Puolakka J, Toivanen J, Suominen H. Effects of hormone replacement therapy and high-impact physical exercise on skeletal muscle in postmenopausal women: A randomized placebo-controlled study. Clin Sci (Lond). 2001; 101(2):147-157 
15. Dobs AS, Nguyen T, Pace C, Roberts CP. Differential effects of oral estrogen versus oral estrogen-androgen replacement therapy on body composition in postmenopausal women. $\mathrm{J}$ Clin Endocrinol Metab. 2002; 87(4):1509-1516.

16. Taaffe DR, Sipila S, Cheng S, Puolakka J, Toivanen J, Suominen H. The effect of hormone replacement therapy and/or exercise on skeletal muscle attenuation in postmenopausal women: A yearlong intervention. Clin Physiol Funct Imaging. 2005; 25(5):297-304.

17. Kenny AM, Kleppinger A, Wang Y, Prestwood KM. Effects of ultra-low-dose estrogen therapy on muscle and physical function in older women. J Am Geriatr Soc. 2005; 53(11):1973-1977.

18. Ribom EL, Piehl-Aulin K, Ljunghall S, Ljunggren O, Naessen T. Six months of hormone replacement therapy does not influence muscle strength in postmenopausal women. Maturitas. 2002; 42(3):225-231.

19. Tanko LB, Movsesyan L, Svendsen OL, Christiansen C. The effect of hormone replacement therapy on appendicular lean tissue mass in early postmenopausal women. Menopause. $2002 ; 9(2): 117-121$

20. Benjamini Y, Hochberg Y. Controlling the false discovery rate: A practical and powerful approach to multiple testing. Journal of the Royal Statistical Society B. 1995; 57(1):289300.

21. Eisen MB, Spellman PT, Brown PO, Botstein D. Cluster analysis and display of genomewide expression patterns. Proc Natl Acad Sci U S A. 1998; 95(25):14863-14868. 
22. Ashburner M, Ball CA, Blake JA, et al. Gene ontology: Tool for the unification of biology. the gene ontology consortium. Nat Genet. 2000; 25(1):25-29.

23. Draghici S, Khatri P, Martins RP, Ostermeier GC, Krawetz SA. Global functional profiling of gene expression. Genomics. 2003; 81(2):98-104.

24. Khatri P, Draghici S, Ostermeier GC, Krawetz SA. Profiling gene expression using ontoexpress. Genomics. 2002; 79(2):266-270.

25. Livak KJ. ABI prism 7700 sequence detection system. user bulletin 2. PE Applied Biosystems. 1997;

26. Liu W, Saint DA. Validation of a quantitative method for real time PCR kinetics. Biochem Biophys Res Commun. 2002; 294(2):347-353.

27. Matthews J, Gustafsson JA. Estrogen signaling: A subtle balance between ER alpha and ER beta. Mol Interv. 2003; 3(5):281-292.

28. Kreppel LK, Blomberg MA, Hart GW. Dynamic glycosylation of nuclear and cytosolic proteins. cloning and characterization of a unique O-GlcNAc transferase with multiple tetratricopeptide repeats. J Biol Chem. 1997; 272(14):9308-9315.

29. Lubas WA, Frank DW, Krause M, Hanover JA. O-linked GlcNAc transferase is a conserved nucleocytoplasmic protein containing tetratricopeptide repeats. J Biol Chem. 1997; 272(14):9316-9324. 
30. Kamemura K, Hart GW. Dynamic interplay between O-glycosylation and Ophosphorylation of nucleocytoplasmic proteins: A new paradigm for metabolic control of signal transduction and transcription. Prog Nucleic Acid Res Mol Biol. 2003; 73:107-136.

31. Whisenhunt TR, Yang X, Bowe DB, Paterson AJ, Van Tine BA, Kudlow JE. Disrupting the enzyme complex regulating O-GlcNAcylation blocks signaling and development. Glycobiology. 2006; 16(6):551-563.

32. Deslypere JP, Young M, Wilson JD, McPhaul MJ. Testosterone and 5 alphadihydrotestosterone interact differently with the androgen receptor to enhance transcription of the MMTV-CAT reporter gene. Mol Cell Endocrinol. 1992; 88(1-3):15-22.

33. Bhasin S, Storer TW, Berman N, Callegari C, Clevenger B, Phillips J, Bunnell TJ, Tricker R, Shirazi A, Casaburi R. The effects of supraphysiologic doses of testosterone on muscle size and strength in normal men. N Engl J Med. 1996; 335(1):1-7.

34. Bhasin S, Woodhouse L, Casaburi R, Singh AB, Mac RP, Lee M, Yarasheski KE, SinhaHikim I, Dzekov C, Dzekov J, Magliano L, Storer TW. Older men are as responsive as young men to the anabolic effects of graded doses of testosterone on the skeletal muscle. J Clin Endocrinol Metab. 2005; 90(2):678-688.

35. Sheffield-Moore M, Paddon-Jones D, Casperson SL, Gilkison C, Volpi E, Wolf SE, Jiang J, Rosenblatt JI, Urban RJ. Androgen therapy induces muscle protein anabolism in older women. J Clin Endocrinol Metab. 2006; 91(10):3844-3849. 
36. Gomes MD, Lecker SH, Jagoe RT, Navon A, Goldberg AL. Atrogin-1, a muscle-specific Fbox protein highly expressed during muscle atrophy. Proc Natl Acad Sci U S A. 2001; 98(25):14440-14445.

37. Bodine SC, Latres E, Baumhueter S, Lai VK, Nunez L, Clarke BA, Poueymirou WT, Panaro FJ, Na E, Dharmarajan K, Pan ZQ, Valenzuela DM, DeChiara TM, Stitt TN, Yancopoulos GD, Glass DJ. Identification of ubiquitin ligases required for skeletal muscle atrophy. Science. 2001; 294(5547):1704-1708.

38. D'Andrea A, Pellman D. Deubiquitinating enzymes: A new class of biological regulators. Crit Rev Biochem Mol Biol. 1998; 33(5):337-352.

39. Quesada V, Diaz-Perales A, Gutierrez-Fernandez A, Garabaya C, Cal S, Lopez-Otin C. Cloning and enzymatic analysis of 22 novel human ubiquitin-specific proteases. Biochem Biophys Res Commun. 2004; 314(1):54-62.

40. Chen YW, Hubal MJ, Hoffman EP, Thompson PD, Clarkson PM. Molecular responses of human muscle to eccentric exercise. J Appl Physiol. 2003; 95(6):2485-2494.

41. Welle S, Brooks AI, Delehanty JM, Needler N, Thornton CA. Gene expression profile of aging in human muscle. Physiol Genomics. 2003; 14(2):149-159.

42. Zambon AC, McDearmon EL, Salomonis N, et al. Time- and exercise-dependent gene regulation in human skeletal muscle. Genome Biol. 2003; 4(10):R61.

43. Welle S, Brooks AI, Delehanty JM, et al. Skeletal muscle gene expression profiles in 20-29 year old and 65-71 year old women. Exp Gerontol. 2004; 39(3):369-377. 
44. Rome S, Clement K, Rabasa-Lhoret R, Loizon E, Poitou C, Barsh GS, Riou JP, Laville M, Vidal H. Microarray profiling of human skeletal muscle reveals that insulin regulates approximately 800 genes during a hyperinsulinemic clamp. J Biol Chem. 2003; 278(20):18063-18068.

45. Giresi PG, Stevenson EJ, Theilhaber J, Koncarevic A, Parkington J, Fielding RA, Kandarian SC. Identification of a molecular signature of sarcopenia. Physiol Genomics. 2005; 21(2):253-263.

46. Timmons JA, Larsson O, Jansson E, Fischer H, Gustafsson T, Greenhaff PL, Ridden J, Rachman J, Peyrard-Janvid M, Wahlestedt C, Sundberg CJ. Human muscle gene expression responses to endurance training provide a novel perspective on duchenne muscular dystrophy. FASEB J. 2005; 19(7):750-760.

47. Teran-Garcia M, Rankinen T, Koza RA, Rao DC, Bouchard C. Endurance training-induced changes in insulin sensitivity and gene expression. Am J Physiol Endocrinol Metab. 2005; 288(6):E1168-78

48. Mahoney DJ, Parise G, Melov S, Safdar A, Tarnopolsky MA. Analysis of global mRNA expression in human skeletal muscle during recovery from endurance exercise. FASEB J. 2005; 19(11):1498-1500.

49. Zahn JM, Sonu R, Vogel H, Crane E, Mazan-Mamczarz K, Rabkin R, Davis RW, Becker KG, Owen AB, Kim SK. Transcriptional profiling of aging in human muscle reveals a common aging signature. PLoS Genet. 2006; 2(7):e115. 
50. Dumeaux V, Johansen J, Borresen-Dale AL, Lund E. Gene expression profiling of wholeblood samples from women exposed to hormone replacement therapy. Mol Cancer Ther. 2006; 5(4):868-876.

51. Edstrom E, Altun M, Hagglund M, Ulfhake B. Atrogin-1/MAFbx and MuRF1 are downregulated in aging-related loss of skeletal muscle. J Gerontol A Biol Sci Med Sci. 2006; 61(7):663-674.

52. Clavel S, Coldefy AS, Kurkdjian E, Salles J, Margaritis I, Derijard B. Atrophy-related ubiquitin ligases, atrogin-1 and MuRF1 are up-regulated in aged rat tibialis anterior muscle. Mech Ageing Dev. 2006.

53. Wells L, Whelan SA, Hart GW. O-GlcNAc: A regulatory post-translational modification. Biochem Biophys Res Commun. 2003; 302(3):435-441.

54. Zhang F, Su K, Yang X, Bowe DB, Paterson AJ, Kudlow JE. O-GlcNAc modification is an endogenous inhibitor of the proteasome. Cell. 2003; 115(6):715-725.

55. Lee DK, Chang C. Endocrine mechanisms of disease: Expression and degradation of androgen receptor: Mechanism and clinical implication. J Clin Endocrinol Metab. 2003; 88(9):4043-4054

56. Hewitt SC, Collins J, Grissom S, Deroo B, Korach KS. Global uterine genomics in vivo: Microarray evaluation of the estrogen receptor alpha-growth factor cross-talk mechanism. Mol Endocrinol. 2005; 19(3):657-668. 
57. Colacurci N, Zarcone R, Mollo A, Russo G, Passaro M, de Seta L, de Franciscis P. Effects of hormone replacement therapy on glucose metabolism. Panminerva Med. 1998; 40(1):1821.

58. Karjalainen A, Paassilta M, Heikkinen J, Backstrom AC, Savolainen M, Kesaniemi YA. Effects of peroral and transdermal oestrogen replacement therapy on glucose and insulin metabolism. Clin Endocrinol (Oxf). 2001; 54(2):165-173.

59. Alonso A, Fernandez R, Moreno M, Ordonez P, Gonzalez-Pardo H, Conejo NM, Diaz F, Gonzalez C. Positive effects of 17 beta-estradiol on insulin sensitivity in aged ovariectomized female rats. J Gerontol A Biol Sci Med Sci. 2006; 61(5):419-426.

60. Ryder JW, Chibalin AV, Zierath JR. Intracellular mechanisms underlying increases in glucose uptake in response to insulin or exercise in skeletal muscle. Acta Physiol Scand. 2001; 171(3):249-257.

61. Godsland IF. Oestrogens and insulin secretion. Diabetologia. 2005; 48(11):2213-2220.

62. McClain DA, Lubas WA, Cooksey RC, Hazel M, parker GJ, Love DC, Hanover JA. Altered glycan-dependent signaling induces insulin resistance and hyperleptinemia. Proc Natl Acad Sci U S A. 2002; 99(16):10695-10699.

63. Wittwer M, Billeter R, Hoppeler H, Fluck M. Regulatory gene expression in skeletal muscle of highly endurance-trained humans. Acta Physiol Scand. 2004; 180(2):217-227. 
64. Rossouw JE, Anderson GL, Prentice RL, et al. Risks and benefits of estrogen plus progestin in healthy postmenopausal women: Principal results from the women's health initiative randomized controlled trial. JAMA. 2002; 288(3):321-333.

65. Veerus P, Hovi SL, Fischer K, Rahu M, Hakama M, Hemminki E. Results from the estonian postmenopausal hormone therapy trial [ISRCTN35338757]. Maturitas. 2006; 55(2):162173. 


\section{TABLES}

Five tables and two supplemental tables are delivered in the separate files to be included into this manuscript.

\section{LEGENDS TO FIGURES}

Figure 1. Flow chart presenting the experimental design and data mining for the genes specifically affected in the non-HRT and HRT groups. Symbols: $\uparrow$ upregulated genes, $\downarrow$ downregulated genes.

Figure 2. Hierarchical clustering of the non-HRT and HRT specific genes. Columns indicate each subject: first five represent non-HRT women followed by 10 HRT subjects. Rows indicate individual genes represented by the difference between follow-up and baseline gene expression values. A) Genes specifically upregulated within non-HRT group. B) Genes specifically downregulated within non-HRT group. C) Genes specifically up- and downregulated within HRT group.

Figure 3. Confirmation of the microarray results by Real Time RT-PCR. Side-by side comparison of change in the gene expression for seven selected genes as determined by Real Time RT-PCR and microarray experiments. For each comparison, mean of change, gene name and Pearson correlation of the results obtained by the two methods (in brackets) are given. Statistical significance: $* * * p \leq 0.001, * * p \leq 0.01$ 